160

\section{MONITORAGGIO MICROBIOLOGICO AMBIENTALE DELLE SALE OPERATORIE DELL'AORN V. MONALDI-NA}

Battista R., Corsi G., Russo F., Bernardo M., Di Fiore G., Smeraglia R.

U.O.C. Microbiologia e Virologia

Direttore Prof. R. Smeraglia

A.O.R.N. V. Monaldi- Napoli

Introduzione. Le sale operatorie (13 nella ns Azienda), classificate ad alto rischio infettivo a carico dei pazienti, sono state monitorate, secondo un programma concordato con il Comitato Infezioni Ospedaliere (CIO), per valutare il grado di contaminazione microbica.

Metodi. Per eseguire una valutazione quantitativa della carica batterica totale mesofila, i controlli sono stati effettuati con metodica di campionamento attivo, utilizzando un campionatore monostadio ad impatto ortogonale: Surface Air System (SAS super 100 PBI) e piastre Rodac (OXOID) contenenti Agar+ neutralizzanti . Il $1^{\circ}$ campionamento dell'aria viene eseguito prima dell'inizio dell'attivita' operatoria, il $2^{\circ}$ all'inizio dell'attivita'. Contemporaneamente al $1^{\circ}$ campionamento vengono eseguiti i controlli delle superfici, utilizzando le stesse piastre Rodac (Oxoid). Prima del $2^{\circ}$ campionamento dell'aria, vengono effettuati i controlli sugli operatori dopo lavaggio chirurgico delle mani. Le piastre vengono incubate a $37^{\circ} \mathrm{C}$ per 24/48 ore. I risultati della lettura sono espressi facendo riferimento ai valori proposti dall'ISPESL.

Risultati.. La lampada scialitica e il pavimento, superfici sulle quali e' stata rilevata piu' frequentemente una contaminazione microbica fuori range, vanno considerate superfici critiche. I controlli delle mani dei 94 operatori sono rientrati tutti nel range di accettabilita'. I controlli dell'aria durante l'attivita' operatoria hanno dato un'incremento della carica batterica in modo direttamente proporzionale al numero di persone presenti in sala.

Conclusioni. L'estrema complessita' dell'attivita' che si svolge in sala operatoria richiede costante attenzione da parte di molteplici figure professionali, dal momento che in tale situazione nulla dovrebbe essere lasciato al caso. Il controllo microbiologico ambientale delle sale operatorie si pone come obiettivo quello di accertare la corretta applicazione delle norme comportamentali adottate dal personale sanitario, e valutare l'efficacia delle procedure di sanificazione messe in atto.

\section{1}

\section{INFEZIONI DELLA FERITA CHIRURGICA IN PAZIENTI ORTOPEDICI: NOSTRA ESPERIENZA}

\author{
Carletti M.', Mascello D. ${ }^{2}$, Di Felice G. ', Carducci G. ', \\ La Rosa G. ${ }^{2}$ \\ 'Laboratorio Analisi, \\ ${ }^{2}$ Ortopedia, Ospedale Pediatrico Bambino Gesù, \\ Presidio di Palidoro
}

Introduzione. Le infezioni della ferita chirurgica rappresentano ancora oggi una complicanza frequente dell'intervento chirurgico, nonostante siano ben conosciute le opportune misure preventive preoperatorie, intraoperatorie e postoperatorie da adottare al fine di ridurne al minimo l'incidenza. In ortopedia l'incidenza di infezioni della ferita chirurgica è bassa se confrontata con quella di altre specialità.

Metodi. Negli anni 2005 e 2006 sono stati inviati 180 tamponi da ferita chirurgica per coltura , su un totale di circa 3185 interventi di chirurgia ortopedica maggiore e minore. I tamponi sono stati seminati sui terreni CNA, MCK, MSA2, SGC2 (Bio Merieux); le identificazioni e gli antibiogrammi sono stati eseguiti mediante sistema automatico Vitek 1 (Bio Merieux).

Risultati. Dai nostri dati emerge che la percentuale di positività si è mantenuta stabile nei due anni (65\% nel 2005 e 69\% nel 2006). Nel 2005 sono stati isolati: 3 Enterococcus faecalis, 2 Providentia stuartii, 23 Staphylococcus aureus, 2 Staphylococcus hominis, 3 Klebsiella pneumoniae, 11 Pseudomonas aeruginosa, 14 Staphylococcus epidermidis, 4 Staphylococcus sciuri. Nel 2006 sono stati isolati:1 Candida albicans, 2 Enterobacter cloacae, 3 Escherichia coli, 5 Proteus mirabilis, 19 Staphylococcus aureus, 1 Staphylococcus hominis, 2 Corynebacterium xerosis, 3 Enterobacter faecalis, 4 Klebsiella pneumoniae, 1 Pseudomonas fluorescens, 12 Staphylococcus epidermidis, 5 Staphylococcus sciuri. Abbiamo preso in considerazione il profilo di antibioticoresistenza dello $\mathrm{S}$. aureus ed è risultato un significativo aumento di resistenza nei confronti dell'oxacillina (da 17\% a $35 \%$ ) e dell'eritromicina (dal $9 \%$ a 35\%).

Conclusioni. I nostri risultati confermano i dati riportati in letteratura: i microrganismi più frequentemente isolati sono aerobi gram positivi, soprattutto S. aureus e stafilococchi coagulasi negativi che mostrano un'aumento di resistenza nei confronti dei comuni antibiotici, in particolare dell'oxacillina. 